\title{
Optical third-harmonic spectroscopy of the magnetic semiconductor EuTe
}

\author{
M. Lafrentz, ${ }^{1}$ D. Brunne, ${ }^{1}$ B. Kaminski, ${ }^{1}$ V. V. Pavlov, ${ }^{2}$ A. B. Henriques, ${ }^{3}$ R. V. Pisarev, ${ }^{2}$ D. R. Yakovlev, ${ }^{1,2}$ G. Springholz, ${ }^{4}$ \\ G. Bauer, ${ }^{4}$ E. Abramof, ${ }^{5}$ P. H. O. Rappl, ${ }^{5}$ and M. Bayer ${ }^{1}$ \\ ${ }^{1}$ Experimentelle Physik 2, Technische Universität Dortmund, 44221 Dortmund, Germany \\ ${ }^{2}$ Ioffe Physical-Technical Institute, Russian Academy of Sciences, 194021 St. Petersburg, Russia \\ ${ }^{3}$ Instituto de Física, Universidade de São Paulo, 05315-970 São Paulo, SP, Brazil \\ ${ }^{4}$ Institute für Halbleiter- und Festkörperphysik, Johannes Kepler Universität Linz, 4040 Linz, Austria \\ ${ }^{5}$ LAS-INPE, 12227-010 São José dos Campos, SP, Brazil
}

(Received 2 August 2010; revised manuscript received 12 October 2010; published 13 December 2010)

\begin{abstract}
EuTe possesses the centrosymmetric crystal structure $m 3 m$ of rocksalt type in which the second-harmonic generation is forbidden in electric dipole approximation but the third-harmonic generation (THG) is allowed. We studied the THG spectra of this material and observed several resonances in the vicinity of the band gap at 2.2-2.5 eV and at higher energies up to $4 \mathrm{eV}$, which are related to four-photon THG processes. The observed resonances are assigned to specific combinations of electronic transitions between the ground $4 f^{7}$ state at the top of the valence band and excited $4 f^{6} 5 d^{1}$ states of $\mathrm{Eu}^{2+}$ ions, which form the lowest energy conduction band. Temperature, magnetic field, and rotational anisotropy studies allowed us to distinguish crystallographic and magnetic-field-induced contributions to the THG. A strong modification of THG intensity for the $2.4 \mathrm{eV}$ band and suppression of the THG for the $3.15 \mathrm{eV}$ band was observed in applied magnetic field. Two main features of the THG spectra were assigned to $5 d\left(t_{2 g}\right)$ and $5 d\left(e_{g}\right)$ subbands at $2.4 \mathrm{eV}$ and $3.15 \mathrm{eV}$, respectively. A microscopic quantum-mechanical model of the THG response was developed and its conclusions are in qualitative agreement with the experimental results.
\end{abstract}

DOI: 10.1103/PhysRevB.82.235206

PACS number(s): 75.50.Pp, 42.65.Ky

\section{INTRODUCTION}

Europium chalcogenides $\mathrm{Eu} X(X=\mathrm{O}, \mathrm{S}, \mathrm{Se}$, and $\mathrm{Te})$ are a group of magnetic semiconductors which possess unique magnetic, transport, and magneto-optical properties determined by the strongly localized $4 f^{7}$ electrons of the $\mathrm{Eu}^{2+}$ ions with spin $S=7 / 2 \cdot{ }^{1-3}$ Due to competition between the exchange integrals of the nearest neighbors (NNs) and nextnearest neighbors (NNNs) their magnetic phase diagrams can include antiferromagnetic (AFM), ferrimagnetic (FIM), and ferromagnetic (FM) ordering as well as a paramagnetic phase. Current interest in $\mathrm{Eu} X$ magnetic semiconductors arises from potential applications for spintronics and magneto-optical devices. ${ }^{4,5}$ Their nonlinear optical and magneto-optical properties, however, remained unexplored until recently when magnetic-field-induced second-harmonic generation (SHG) was observed in EuTe, EuSe, ${ }^{6,7}$ and in $\mathrm{EuO}^{8}{ }^{8}$

Nonlinear optics is a large, continuously expanding field of fundamental and applied research. Important phenomena in this field are related with the electric dipole (ED) induced conversion of the frequency $\omega$ of a fundamental light field $E(\omega)$. Examples are sum and difference frequency generation, higher-order harmonics generation, parametric amplification, stimulated Raman scattering, and many others. In these processes a single light field $E(\omega)$ is involved in nonlinear optical interactions determined by different selection rules, e.g., compared with linear optics, and, therefore, opening novel experimental possibilities.

Among a vast variety of nonlinear phenomena related with frequency conversion the SHG and third-harmonic generation (THG) are the simplest processes. The relevant nonlinear polarization $\mathbf{P}(2 \omega, 3 \omega)$ can be written as ${ }^{9,10}$

$$
P_{i}(2 \omega, 3 \omega)=\chi_{i j k}^{(2)} E_{j}(\omega) E_{k}(\omega)+\chi_{i j k l}^{(3)} E_{j}(\omega) E_{k}(\omega) E_{l}(\omega),
$$

where $\chi_{i j k}^{(2)}$ and $\chi_{i j k l}^{(3)}$ are the second-order and third-order nonlinear optical susceptibilities, responsible for the crystallographic contributions to $\mathrm{SHG}$ and $\mathrm{THG}$, respectively. $E_{j, k, l}(\omega)$ are the fundamental optical fields. Parity restrictions immediately imply that in the ED approximation the SHG process, described by a polar third-rank tensor $\chi_{i j k}^{(2)}$, is only allowed in noncentrosymmetric media. Those can be media with broken space-inversion symmetry operation, e.g., at structural or magnetic phase transitions. ${ }^{11}$ This explains why the majority of SHG research in solids is focused on piezoelectric and ferroelectric materials oriented to practical applications in nonlinear optics. ${ }^{12}$

By contrast the THG process described by a polar fourthrank tensor $\chi_{i j k l}^{(3)}$ is allowed in materials of any symmetry. ${ }^{910,13,14}$ Being described by a higher-order nonlinear susceptibility the THG is much weaker than the SHG in media where SHG is allowed in the electric dipole approximation. However, as THG is allowed in any medium it can be used for investigating materials where SHG is forbidden. Also the strong increase in the susceptibility in the vicinity of electronic resonances can provide larger intensities of THG. Further, involvement of high-order nonlinearities into THG allows one to study hidden symmetries modified by phase transitions or external field symmetry breaking. ${ }^{15}$

The numerous THG studies have covered gases, plasmas, liquids, liquid crystals, solids, photonic crystals, and metamaterials. Some examples of the THG in magnetically ordered materials can be found in Refs. 16-22. THG processes can be used as a tool for microscopical imaging of small structures such as clusters, domains and domain walls, ${ }^{23}$ dynamical objects, ${ }^{24}$ and can be efficiently used as 
nondestructive diagnostic tool for biological objects. ${ }^{25,26}$

New pathways may be opened when THG is studied in external electric or magnetic fields, which reduce the symmetry of an object under study. In those cases when both the SHG and THG processes are allowed in the same medium they can provide supplementary information on crystallographic, electronic, and magnetic structures due to the different selection rules of these mutually complementary processes.

Applying the restrictions imposed by Eq. (1) to the europium chalcogenides with centrosymmetric crystal structure we immediately see that ED-SHG is forbidden but ED-THG is allowed. This is encouraging for experimental and theoretical studies of THG in this material class. Our study is motivated by the fact that the potential of THG nonlinear spectroscopy has remained rather unexplored. The overwhelming majority of THG studies exploited single laser wavelengths or scanned narrow spectral ranges. Only a spectroscopic approach, as demonstrated by recent SHG studies, $6,11,27,28$ allows one to draw unambiguous conclusions on the microscopic nature of the observed nonlinear signals. In particular, THG spectroscopy with high spectral resolution at low temperatures remains to be applied to semiconductors and magnetic materials. The few examples of THG studies in semiconductors were performed at fixed laser wavelengths. ${ }^{29,30}$

In this paper we report on a spectroscopic study of crystallographic and magnetic-field-induced THG in the magnetic semiconductor EuTe. We studied the THG spectra in the vicinity of the band gap at $2.2-2.5 \mathrm{eV}$ and at higher energies up to $4 \mathrm{eV}$. The spectroscopic study of the THG was performed as a function of temperature and magnetic field. A strong modification of the THG at $2.4 \mathrm{eV}$ and suppression of the THG at $3.15 \mathrm{eV}$ in applied magnetic field was revealed by temperature and rotational anisotropy studies. A quantummechanical model of THG was developed and its conclusions are in qualitative agreement with the experimental results. It was found that two main features of the THG at 2.4 $\mathrm{eV}$ and $3.15 \mathrm{eV}$ can be assigned to $5 d\left(t_{2 g}\right)$ and $5 d\left(e_{g}\right)$ subbands in EuTe, respectively.

Paper is organized as follows. In Sec. II we briefly describe the crystal and electronic structures, as well as the magnetic, optical, and magneto-optical properties of EuTe. Section III gives the symmetry description of crystallographic and magnetic-field-induced THG in EuTe. Experimental results are presented in Sec. IV. Section V is devoted to the theoretical model and to its comparison with experimental data. Section VI concludes the paper.

\section{PROPERTIES OF EUROPIUM CHALCOGENIDES}

The europium chalcogenides $\operatorname{Eu} X(X=\mathrm{O}, \mathrm{S}, \mathrm{Se}$, and $\mathrm{Te})$ are wideband-gap magnetic semiconductors, characterized by several unique properties. For many years they have attracted interest. For example, EuO has been suggested for applications in spin-filter devices, ${ }^{5,31-34}$ and EuS (Refs. 35-41) as well as EuSe (Ref. 42) for tunnel junctions. Recently it was shown, that EuO can be epitaxially grown on $\mathrm{Si}$ and $\mathrm{GaN}$, which opens new possibilities for device realization. ${ }^{4,43}$



FIG. 1. (Color online) Crystal structure of EuTe, spheres with arrows show the $\mathrm{Eu}^{2+}$ and spheres without show $\mathrm{Te}^{2-}$ ions. Gray triangle indicates the (111) plane with parallel alignment of the $\mathrm{Eu}^{2+}$ spins below the Néel temperature $T_{N}=9.58 \mathrm{~K}$. Spins of neighboring planes are aligned antiferromagnetically.

\section{A. Crystallographic structure}

The europium chalcogenides crystallize in the centrosymmetric cubic rocksalt structure with $m 3 m\left(O_{h}\right)$ symmetry. Figure 1 shows the crystal structure of EuTe with a lattice constant $a_{0}=6.598 \AA$. The face-centered-cubic lattice contains a two-atomic basis with a $\mathrm{Eu}^{2+}$ ion at $(1 / 2,1 / 2,1 / 2)$ and a $\mathrm{Te}^{2-}$ ion at $(0,0,0)$. The divalent $\mathrm{Eu}^{2+}$ ions are octahedrally surrounded by six $\mathrm{Te}^{2-}$ ions forming a strong ionic binding, which results in empty $5 d$ states of europium and completely filled $p$ orbitals of tellurium.

\section{B. Electronic band structure}

The electronic level structure of the europium chalcogenides is of key importance for understanding their magnetic, optical, and magneto-optical properties. The electronic properties are determined by the $4 f^{7}$ electrons of the $\mathrm{Eu}^{2+}$ ions with spin $S=7 / 2$ and the electrons of the chalcogens. ${ }^{1,44,45}$ It is generally accepted that the $4 f^{7}$ atomic states of $\mathrm{Eu}^{2+}$ constitute the valence level in $\mathrm{Eu} X$, which lies within the energy gap, directly on top of a $2-3 \mathrm{eV}$ wide valence band formed by the $n p$ orbitals of the chalcogen, where $n=2,3,4$, and 5 for $\mathrm{O}, \mathrm{S}, \mathrm{Se}$, and Te, respectively. The conduction band can be described by a tight-binding model, in which the basis wave functions consist of the $5 d$ orbitals of the $\mathrm{Eu}^{2+}$ ions, split by the octahedral crystal field into a twofold degenerate low-energy $5 d\left(t_{2 g}\right)$ state and a $5 d\left(e_{g}\right)$ state at about $1 \mathrm{eV}$ higher energy. These assertions are consistent with magneto-optical data. ${ }^{46}$ It is also generally assumed that a broad conduction band with $6 s$ character overlaps with the $5 d\left(t_{2 g}\right)$ states. However, electric dipole coupling of the $4 f^{7}\left({ }^{8} S_{7 / 2}\right)$ state to this $6 s$ band is forbidden, which is therefore optically dark. The single-particle electronic structure of EuTe is shown schematically in Fig. 2.

The $4 f^{7}$ electrons are strongly localized around the $\mathrm{Eu}^{2+}$ ion to which they belong, because of the Coulomb attraction by the unscreened $\mathrm{Eu}$ core, and they are screened from the surrounding by electrons occupying the $5 s$ and $5 p$ orbitals of the $\mathrm{Eu}^{2+}$ ion. Therefore the $4 f^{7}$ electrons are unaffected by the crystal environment and can be described by the wave 




FIG. 2. (Color online) Scheme of the energy band structure of EuTe. For single photon processes in the vicinity of the band gap only ED transitions are allowed. Open dots represent the imaginary part of the dielectric function, taken from Ref. 2.

functions of an isolated $\mathrm{Eu}$ atom. The standard spectroscopic notation of this state is, therefore, $4 f^{7}\left({ }^{8} S_{7 / 2}\right)$. The excitation of lowest energy corresponds to the transfer of one electron from the $4 f^{7}\left({ }^{8} S_{7 / 2}\right)$ state to the $5 d\left(t_{2 g}\right)$ conduction band. Thereafter only six electrons are left on the $4 f$ orbital so that the excited state is often labeled as $4 f^{6} 5 d^{1}$. Here the upper indices correspond to the number of electrons on corresponding orbitals.

\section{Magnetic properties}

The magnetic properties of the europium chalcogenides are determined by the ground state of the $\mathrm{Eu}^{2+}$ ions in which the $4 f^{7}$ electrons with spin $S=7 / 2$ are involved. ${ }^{1,44,45}$ The $\mathrm{Eu} X$ are classical Heisenberg magnets where the competition between the NN exchange integral $J_{1}$, which provide a ferromagnetic interaction, and the NNN integral $J_{2}$ providing an antiferromagnetic interaction results in magnetic phase diagrams that may include AFM, FIM, and FM ordering, as well as a paramagnetic phase at elevated temperatures in EuTe and EuSe. ${ }^{1,47}$ The unique magnetic properties and complicated magnetic phase diagrams of $\mathrm{Eu} X$ are caused by the varying ratios of the $J_{1}$ and $J_{2}$ integrals and by their competition with an external magnetic field.

In EuTe $\left|J_{2}\right|>\left|J_{1}\right|$ and, therefore, this compound shows antiferromagnetism below the Néel temperature $T_{N}$ $=9.58 \mathrm{~K}^{1}{ }^{1}$ The $\mathrm{Eu}^{2+}$ spins are parallel within the (111) planes and adjacent planes have alternating spin orientations $[\uparrow \downarrow \uparrow \downarrow]$, see Fig. 1. At lattice temperatures $T<2 \mathrm{~K}$ and in magnetic fields exceeding a critical value $H_{C}=7.2 \mathrm{~T}$ the high-field paramagnetic phase is reached, and the magnetization is saturated as it is oriented along the external field.

Below $T_{N}$ the magnetic ordering in EuTe can be characterized by the magnetic moments $\mathbf{m}_{1}$ and $\mathbf{m}_{2}$ of the two sublattices with $\left|m_{1}\right|=\left|m_{2}\right|$. To describe the magnetic behavior of an antiferromagnet in external magnetic field we introduce the FM vector $\mathbf{F}=\mathbf{m}_{1}+\mathbf{m}_{2}$ and the AFM vector $\mathbf{A}$ $=\mathbf{m}_{1}-\mathbf{m}_{2}$, as discussed in Refs. 6 and 7. The external magnetic field induces the axial vector $\mathbf{F}$. From the experimental studies of THG in EuTe, presented in Sec. IV, it is quite obvious that only the ferromagnetic component $\mathbf{F}$ of the magnetic structure below Néel temperature and the fieldinduced magnetization, $\mathbf{M}$, above $T_{N}$ are responsible for the observed anisotropy of the magnetic-field-induced THG. In

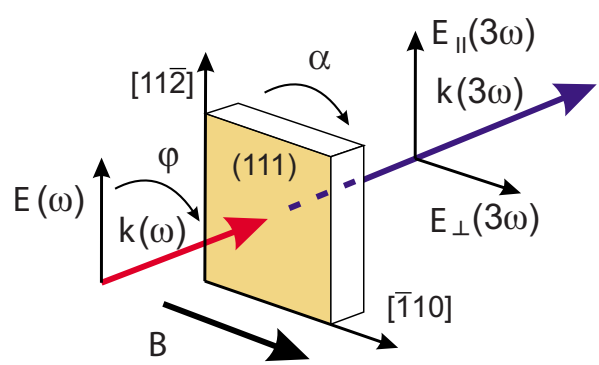

FIG. 3. (Color online) Sketch of experimental geometry in the THG studies for (111)-oriented $\mathrm{Eu} X$ film. $\mathbf{k}(\omega)$ and $\mathbf{k}(3 \omega)$ are light wave vectors for the fundamental and third-harmonic waves, respectively. Azimuthal angle $\varphi$ characterizes the relative orientation of polarization plane of the fundamental wave $\mathbf{E}(\omega)$ and the external magnetic field $\mathbf{B}$ in the Voigt geometry. $\alpha$ is the sample azimuthal angle. Note, in case of (001) orientation of the $\mathrm{EuX}$ film the horizontal and vertical crystal axes are [100] and [010], respectively.

contrast to SHG, antiferromagnetic and paramagnetic spin configurations also make contributions to THG as discussed in Sec. $\mathrm{V}$ and shown in Fig. 12.

\section{Optical and magneto-optical properties}

The optical and magneto-optical properties of the europium chalcogenides are striking, and some of them are unmatched by any other magnetic semiconductor. As discussed above, the optical band gap is formed by the lowest energy excitation corresponding to the transfer of an electron from the $4 f^{7}\left({ }^{8} S_{7 / 2}\right)$ state to the $5 d\left(t_{2 g}\right)$ conduction band. When subject to an external magnetic field, EuTe exhibits a giant low-energy shift of $15 \mathrm{meV} / \mathrm{T}$ of the optical-absorption threshold. ${ }^{1,44}$ The Faraday effect is also extraordinary large with Verdet constants as high as $10^{6} \mathrm{deg} / \mathrm{cm} .{ }^{48-53}$ Due to the giant Faraday rotation EuX films can be applied also for high-resolution magneto-optical imaging of the flux distribution in superconductors. ${ }^{54}$

\section{SYMMETRY CONSIDERATION OF THG IN EUROPIUM CHALCOGENIDES}

The underlying coherent mechanism of harmonics generation are multiphoton processes which become strongly anisotropic even in cubic crystals such as the $\mathrm{EuX}$. A particularly characteristic property of harmonics generation is the rotational anisotropy which reflects the changes in the harmonic intensity as a function of the azimuthal angle of the incident and generated light polarizations. We discuss in this section the symmetry properties and rotational anisotropies of crystallographic and magnetic-field-induced THG in $\mathrm{EuX}$.

\section{A. Crystallographic THG}

First we analyze the expected ED nonlinearity of third order in the $\operatorname{Eu} X$ compounds. The basic geometry for the nonlinear optical THG experiments is shown in Fig. 3.

In the ED approximation the crystallographic (CED) third-order nonlinear optical polarization $P_{i}^{C E D}(3 \omega)$ can be written as ${ }^{9,10}$ 


$$
P_{i}^{\mathrm{CED}}(3 \omega)=\chi_{i j k l}^{(3)} E_{j}(\omega) E_{k}(\omega) E_{l}(\omega),
$$

where $\chi_{i j k l}^{(3)}$ is the third-order nonlinear optical susceptibility responsible for the crystallographic part of the THG. The polar fourth-rank tensor $\chi_{i j k l}^{(3)}$ is nonzero in crystals of any symmetry and the relevant nonvanishing tensor components are (independent components are underlined), ${ }^{13,14}$

$$
\begin{gathered}
\underline{\chi_{x y y x}}=\chi_{x z z x}=\chi_{y x x y}=\chi_{y z z y}=\chi_{z x x z}=\chi_{z y y z}, \\
\underline{\chi_{x y x y}}=\chi_{x z x z}=\chi_{y x y x}=\chi_{y z y z}=\chi_{z x z x}=\chi_{z y z y}, \\
\underline{\chi_{x x y y}}=\chi_{x x z z}=\chi_{y y x x}=\chi_{y y z z}=\chi_{z z x x}=\chi_{z z y y}, \\
\underline{\chi_{x x x x}}=\chi_{y y y y}=\chi_{z z z z} .
\end{gathered}
$$

Due to permutability of the last three indices of $\chi_{i j k l}^{(3)}$, which arises from the permutation symmetry of the three incident optical fields $E_{j, k, l}(\omega)$, one can show that $\chi_{x x y y}=\chi_{x y x y}=\chi_{x y y x}$. As a result, the number of independent components is reduced to two, namely, $\chi_{x x y y}$ and $\chi_{x x x x}$. Therefore, we can write down the nonlinear polarization $P_{\|}^{\mathrm{CED}}(3 \omega)$ for the parallel configuration $\mathbf{E}(3 \omega) \| \mathbf{E}(\omega)$ in case of $\mathbf{k}(\omega) \|[111]$ as

$$
P_{\|}^{\mathrm{CED}}(3 \omega)=\frac{1}{2}\left(\chi_{x x x x}+3 \chi_{x x y y}\right) .
$$

This means that the THG does not depend on the azimuthal angle $\varphi$ in the used geometry and shows an isotopic behavior for rotation of the light polarization plane with respect to the crystallographic axes. For the crossed configuration $\mathbf{E}(3 \omega) \perp \mathbf{E}(\omega), \mathbf{k}(\omega) \|[111]$ one obtains

$$
P_{\perp}^{\mathrm{CED}}(3 \omega)=0 \text {. }
$$

Therefore, for the $\mathbf{k}(\omega) \|[111]$ geometry isotropic THG signals can appear only for the parallel configuration of $\mathbf{E}(3 \omega)$ and $\mathbf{E}(\omega)$.

In the case of a crystal orientation $\mathbf{k}(\omega) \|[001]$ the nonlinear polarization $P_{\|}^{\mathrm{CED}}(3 \omega)$ can be written for the parallel configuration $\mathbf{E}(3 \omega) \| \mathbf{E}(\omega)$ as

$$
\begin{aligned}
P_{\|}^{\mathrm{CED}}(3 \omega)= & \frac{1}{4}\left[3\left(\chi_{x x x x}+\chi_{x x y y}\right)+\left(\chi_{x x x x}-3 \chi_{x x y y}\right)\right. \\
& \times \cos (4 \varphi-4 \alpha)] .
\end{aligned}
$$

For the crossed configuration $\mathbf{E}(3 \omega) \perp \mathbf{E}(\omega), \mathbf{k}(\omega) \|[001]$ one can write

$$
P_{\perp}^{\mathrm{CED}}(3 \omega)=\frac{1}{4}\left(3 \chi_{x x y y}-\chi_{x x x x}\right) \sin (4 \varphi-4 \alpha) .
$$

Thus, Eqs. (6) and (7) demonstrate that the coherent multiphoton THG process is strongly anisotropic in cubic (001)oriented $\mathrm{Eu} X$ crystals.

\section{B. Magnetic-field-induced THG}

Now we analyze the expected ED nonlinearity of fourth order in the $\mathrm{Eu} X$ compounds in presence of an external magnetic field in the Voigt geometry. In ED approximation one can write the magnetic-field-induced (IED) nonlinear polarization $P_{i}^{\mathrm{IED}}(3 \omega)$ in the following form:

$$
P_{i}^{\mathrm{IED}}(3 \omega)=\chi_{i j k l m}^{(4)} E_{j}(\omega) E_{k}(\omega) E_{l}(\omega) M_{m}(0) .
$$

The THG susceptibility $\chi_{i j k l m}^{(4)}$ is an axial fifth-rank tensor ${ }^{13}$ and $M_{m}(0)$ is a component of the axial magnetization vector. Below $T_{N}$ this vector coincides with the ferromagnetic vector $\mathbf{F}$ while above $T_{N}$ it is identical with the field-induced magnetization in the paramagnetic phase. Within ED approximation this THG contribution is allowed in crystals of any symmetry. The nonvanishing tensor components $\chi_{i j k l m}^{(4)}$ can be found in Ref. 13. The second, third, and fourth indices of the susceptibility $\chi_{i j k l m}^{(4)}$ denote the direction of the incident photons, which are indistinguishable. Thus these indices have to be permutable. Due to this permutability only three independent (underlined) components remain

$$
\begin{aligned}
& \underline{\chi_{z y y x y}}=\chi_{x z z y z}=-\chi_{z x x y x}=\chi_{y x x z x}=-\chi_{y z z x z}=-\chi_{x y y z y}, \\
& \underline{\chi_{z y y y x}}=\chi_{x z z z y}=-\chi_{z x x x y}=\chi_{y x x x z}=-\chi_{y z z z x}=-\chi_{x y y y z}, \\
& \underline{\chi_{z z z y x}}=\chi_{x x x z y}=-\chi_{z z z x y}=\chi_{y y y x z}=-\chi_{y y y z x}=-\chi_{x x x y z} .
\end{aligned}
$$

These components lead to the following anisotropies of the magnetic-field-induced nonlinear optical polarization $P_{\|}^{\mathrm{IED}}(3 \omega)$ for $\mathbf{E}(3 \omega) \| \mathbf{E}(\omega)$ and $\mathbf{k}(\omega) \|[111]$,

$$
\begin{aligned}
P_{\|}^{\mathrm{IED}}(3 \omega)= & \frac{\sqrt{2}}{12}\left(\chi_{z z z y x}-\chi_{z y y y x}\right)[\cos (2 \varphi-3 \alpha) \\
& +\cos (4 \varphi-3 \alpha)],
\end{aligned}
$$

and for the crossed configuration $\mathbf{E}(3 \omega) \perp \mathbf{E}(\omega)$ and $\mathbf{k}(\omega) \|[111]$,

$$
\begin{aligned}
P_{\perp}^{\mathrm{IED}}(3 \omega)= & \frac{\sqrt{2}}{12}\left[\left(2 \chi_{z y y x y}+\chi_{z z z y x}+\chi_{z y y y x}\right) \sin (2 \varphi-3 \alpha)\right. \\
& \left.+\left(\chi_{z z z y x}-\chi_{z y y y x}\right) \sin (4 \varphi-3 \alpha)\right]
\end{aligned}
$$

For (001) orientation with $\mathbf{k}(\omega) \|[001]$ the nonlinear polarization $P_{\|}^{\mathrm{IED}}(3 \omega)$ for the parallel configuration $\mathbf{E}(3 \omega) \| \mathbf{E}(\omega)$ results in

$$
P_{\|}^{\mathrm{IED}}(3 \omega)=0
$$

and for the crossed configuration $\mathbf{E}(3 \omega) \perp \mathbf{E}(\omega)$ as well in

$$
P_{\perp}^{\mathrm{IED}}(3 \omega)=0 .
$$

In the absorption region related to the electronic transition $4 f^{7} \rightarrow 4 f^{6} 5 d^{1}$, the tensors $\chi_{i j k l}^{(3)}$ and $\chi_{i j k l m}^{(4)}$ are complex. So for the THG intensity the interference between IED and CED contributions should be taken into account by

$$
I(3 \omega) \propto\left|\mathbf{P}^{\mathrm{CED}}\right|^{2}+\left|\mathbf{P}^{\mathrm{IED}}\right|^{2} \pm 2\left|\mathbf{P}^{\mathrm{CED}}\right|\left|\mathbf{P}^{\mathrm{IED}}\right| \cos \phi
$$

with $\phi$ as a relative phase between IED and CED contributions. Here the first term is a pure crystallographic contribution. The second term is a pure magnetic-field-induced contribution, which is proportional to $M^{2}$. The third interference term is proportional to the axial vector $\mathbf{M}$, where \pm signs refer to opposite orientations of $\mathbf{M}$. Neglecting energy dissipation in the medium, $\chi_{i j k l}^{(3)}$ is a real tensor and $\chi_{i j k l m}^{(4)}$ is a pure 
imaginary tensor and the interference term does not appear as $\phi=90^{\circ}$. In the case of energy dissipation or presence of resonance states the interference term can appear similar to the interference of crystallographic and magnetic contributions in the SHG process. ${ }^{11,55} \mathrm{~A}$ microscopic quantummechanical model, developed in Sec. V, considers a general third order polarization for the IED and CED contributions, therefore the phase $\phi$ is preserved for the entire spectral range and the interference term in Eq. (14) is neglected. However, this model takes into account the interference between the third-order polarizations coming from different magnetic sublattices in EuTe.

\section{EXPERIMENTAL RESULTS}

\section{A. Experiment}

The THG experiments were performed on an EuTe epilayer of $1.5 \mu \mathrm{m}$ thickness grown by molecular-beam epitaxy (MBE) on (111)-oriented $\mathrm{BaF}_{2}$ substrates. ${ }^{56,57}$ The EuTe layer was capped with a $0.2-\mu \mathrm{m}$-thick $\mathrm{BaF}_{2}$ protective layer. The high structural sample quality was confirmed by $\mathrm{x}$-ray analysis. THG was studied using the experimental technique described in Refs. 27 and 28. THG spectra were recorded in transmission geometry using $8 \mathrm{~ns}$ light pulses with a $10 \mathrm{~Hz}$ repetition rate, generated by an optical parametric oscillator (OPO) pumped by the third harmonic of a solid-state $\mathrm{Nd}$ : yttrium aluminum garnet laser $(355 \mathrm{~nm})$. A tunable type-II OPO allowed us to access a wide spectral range (410-2500 $\mathrm{nm}$ ) with a typical pulse power of $20 \mathrm{~mJ}$ and a narrow linewidth of about $0.4 \mathrm{meV}$. A Glan-Thomson prism and quarterand/or half-wave plates were used to set the required light polarization at the fundamental frequency. The laser light beam of the OPO was directed onto the sample and the resulting THG signal was analyzed by polarization optics. The signal was dispersed by a $0.5 \mathrm{~m}$ spectrometer and detected by a cooled charge-coupled-device camera. The sample temperature was varied from 5 to $300 \mathrm{~K}$. Magnetic fields up to 7 $\mathrm{T}$ were applied in the Voigt geometry perpendicular to both the layer growth axis [111] and the light wave vector.

\section{B. Crystallographic contribution}

Figure 4 shows THG spectra of EuTe recorded at $T$ $=5 \mathrm{~K}$ for $\mathbf{E}(3 \omega) \| \mathbf{E}(\omega)$ and $\mathbf{E}(3 \omega) \perp \mathbf{E}(\omega)$ over the broad spectral range from 2.1 up to $3.9 \mathrm{eV}$. No signal could be detected in $\mathbf{E}(3 \omega) \perp \mathbf{E}(\omega)$ configuration, which is in agreement with the symmetry considerations for the CED contributions, see Eq. (5). In the $\mathbf{E}(3 \omega) \| \mathbf{E}(\omega)$ configuration, where the CED contribution is symmetry allowed, a strong THG signal was detected consisting of four bands at 2.4, 2.75, 3.15 , and $3.7 \mathrm{eV}$. All these bands have an isotropic rotational diagram, which is exemplified in the inset of Fig. 4 for the energy of $3.15 \mathrm{eV}$. Such diagram is characteristic for the CED contribution of (111)-oriented EuTe, see Eq. (4).

Increase of the lattice temperature up to $300 \mathrm{~K}$ does not change significantly the THG spectrum. As one can see in Fig. 4, the spectral position, the broadening, and the intensity of THG bands are very stable with respect to increasing the temperature up to $200 \mathrm{~K}$. Even further increase up to $300 \mathrm{~K}$

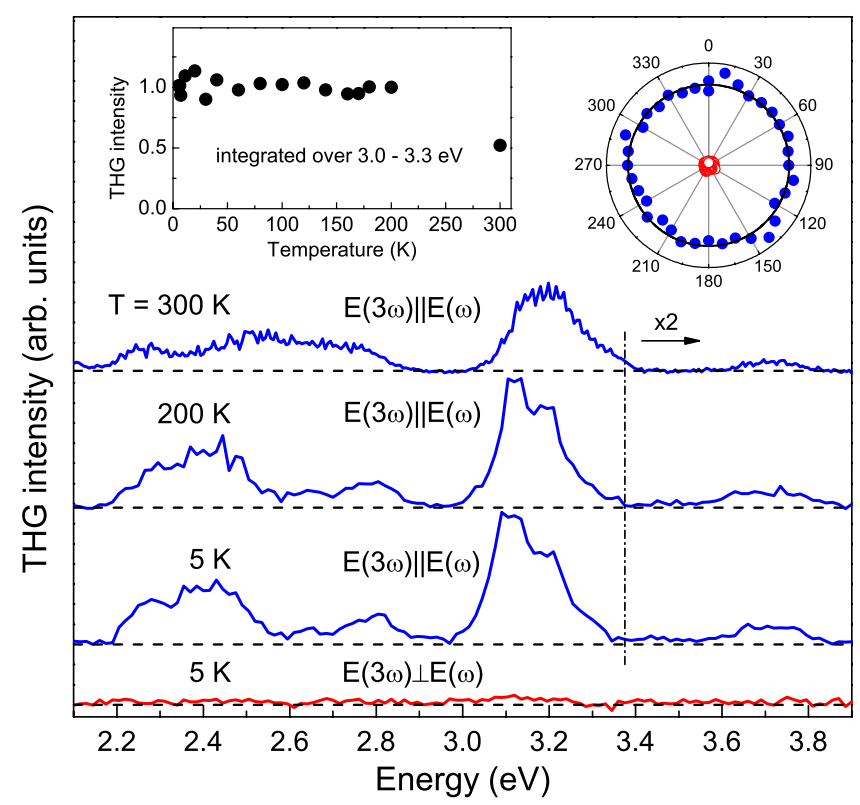

FIG. 4. (Color online) THG spectra of EuTe for two experimental geometries and for $T=5,200$, and $300 \mathrm{~K}$. Dotted lines indicate zero level. Signal intensities above $3.4 \mathrm{eV}$ are multiplied by a factor of 2. Insets show the temperature dependence of the THG intensity integrated in the spectral range from $3.0-3.3 \mathrm{eV}$ and the angular dependence of the THG intensity at $3 \hbar \omega=3.15 \mathrm{eV}$ and $T=5 \mathrm{~K}$. In the rotational diagram symbol color indicates the configuration: blue closed circles located in vicinity of a black solid line for $\mathbf{E}(3 \omega) \| \mathbf{E}(\omega)$ and red open circles in the very center for $\mathbf{E}(3 \omega) \perp \mathbf{E}(\omega)$.

does not bring strong spectral modifications and causes only a twofold decrease in the THG intensity. The temperature dependence of the THG intensity integrated over the strongest line in the range $3.0-3.3 \mathrm{eV}$ is given in the inset. Such a temperature dependence together with the isotropic rotational diagram allows us to conclude that at zero magnetic field the THG spectra do not contain magnetic contributions.

Let us now assign the THG bands to the electronic structure of EuTe, which is schematically shown in Fig. 5. The band at $2.4 \mathrm{eV}$ corresponds to the $4 f^{7} \rightarrow 4 f^{6} 5 d^{1}\left(t_{2 g}\right)$ optical transition in the EuTe band gap vicinity. It was also observed in SHG and optical-absorption spectra. ${ }^{7,57}$ Another band at $3.15 \mathrm{eV}$ is related to the $4 f^{7} \rightarrow 4 f^{6} 5 d^{1}\left(e_{g}\right)$ transition. The high-energy band at $3.7 \mathrm{eV}$ can be assigned to a two-photon process at the $4 f^{7} \rightarrow 4 f^{6} 5 d^{1}\left(t_{2 g}\right)$ optical transition, i.e., the $4 f^{6} 5 d^{1}\left(t_{2 g}\right)$ state acts as an intermediate resonance state for the THG process, whose final energy falls into the continuum of the $5 d$ and $6 s$ states.

\section{Magnetic-field-induced contribution}

As we have seen in Sec. III, the crystallographic contribution to the THG signal vanishes in $\mathbf{E}(3 \omega) \perp \mathbf{E}(\omega)$ configuration while the symmetry consideration predicts that the magnetic-field-induced contribution should be observable in this configuration, see Eq. (11). Therefore, this configuration is convenient to study the properties of the purely magnetic contribution. 


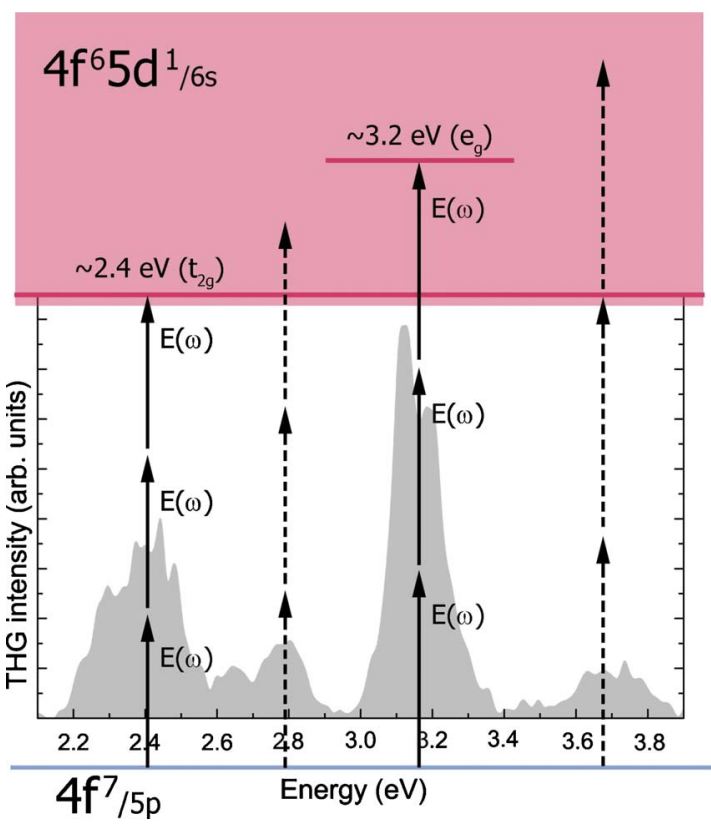

FIG. 5. (Color online) Scheme of the THG processes in EuTe. The THG spectrum shown by gray area is the one from Fig. 4 measured at $T=5 \mathrm{~K}$ in $\mathbf{E}(3 \omega) \| \mathbf{E}(\omega)$ configuration. Four bands at $2.4,2.75,3.15$, and $3.7 \mathrm{eV}$ are detected experimentally. Two more intense THG bands at 2.4 and $3.15 \mathrm{eV}$ are assigned to $5 d\left(t_{2 g}\right)$ and $5 d\left(e_{g}\right)$ subbands in EuTe for four-photon processes.

In Fig. 6(a) the THG spectra measured in magnetic fields of 0 and $7 \mathrm{~T}$ in $\mathbf{E}(3 \omega) \perp \mathbf{E}(\omega)$ configuration are shown. One sees a strong band at $2.43 \mathrm{eV}$ with an anisotropic rotational diagram as expected for the magnetic-field-induced IED contribution, see Eq. (11). Other bands are not observed in the spectral range from 2.6 to $3.9 \mathrm{eV}$ due to relatively weak overall signal intensity. The magnetic-field dependence of the THG intensity for the band at $2.43 \mathrm{eV}$ in Fig. 6(b) shows a monotonous increase with a trend of saturation in strong magnetic fields. This arises from the behavior of the ferromagnetic vector $\mathbf{F}$ and is similar to the magnetic-field dependence of the SHG intensity in EuTe, shown in Fig. 7 of Ref. 7. As expected, the magnetic-field-induced contribution has a strong temperature dependence reflecting the decrease in the magnetic susceptibility in the paramagnetic phase with increasing lattice temperature. This dependence is presented in Fig. 6(c). Note that the THG intensity vanishes for $T$ $>30 \mathrm{~K}$, which is in strong contrast with the properties of the crystallographic contribution shown in Fig. 4.

Both crystallographic and magnetic-field-induced contributions are allowed for $\mathbf{E}(3 \omega) \| \mathbf{E}(\omega)$ configuration, for which spectra at different magnetic fields are shown in Fig. 7 (a). Whereas the intensity of the $2.4 \mathrm{eV}$ band grows with increasing magnetic field, the intensities of the other bands at $2.75,3.15$, and $3.7 \mathrm{eV}$ show a decreasing behavior in magnetic fields exceeding $4 \mathrm{~T}$. These magnetic-field dependencies are given in the inset of Fig. 7(a).

The rotational diagrams measured for the three bands with and without magnetic field are given in Fig. 7(b). In absence of the external magnetic field all three diagrams are isotropic, see closed circles for experimental data and gray areas for the fit by Eq. (4). In a magnetic field of $7 \mathrm{~T}$ the diagrams


FIG. 6. (Color online) (a) THG spectra of EuTe measured in the $\mathbf{E}(3 \omega) \perp \mathbf{E}(\omega) \perp \mathbf{B}(0)$ configuration in magnetic fields of 0 and $7 \mathrm{~T}$. Dashed lines indicate zero level. The inset shows rotational diagram measured at $3 \hbar \omega=2.43 \mathrm{eV}$ at $B=7 \mathrm{~T}$. (b) Magnetic-field dependence of the THG intensity integrated over the spectral range from 2.4 to $2.5 \mathrm{eV}$. (c) Temperature dependence of the integrated THG intensity at $B=4 \mathrm{~T}$.

become anisotropic, see open circles, in accord with predictions of Eq. (10).

Figure 8 shows THG spectra for positive and negative values of magnetic fields of $\pm 7 \mathrm{~T}$ in the spectral range of 2.2-3.4 eV. A strong modification of THG intensity for the $2.4 \mathrm{eV}$ band is observed, whereas the $3.15 \mathrm{eV}$ band is insensitive to the inversion of the magnetic-field direction. A behavior of the $2.4 \mathrm{eV}$ band can be understood, when taking into account the interference between CED and IED, as considered in Sec. III. The change in magnetic-field sign produces opposite orientations of $\mathbf{M}$, therefore, the total THG intensity is changed due to the constructive or distractive interference of the CED and IED contributions in accordance with Eq. (14). However, the interference does not take place for $2.75,3.15$, and $3.7 \mathrm{eV}$ bands and their decrease in applied magnetic field can be attributed to the second-order processes in respect to the axial vector $\mathbf{M}$. These processes are not included in the macroscopic and microscopic considerations in this paper and require further experimental and theoretical studies.

\section{Comparison of THG and SHG spectra}

Though THG and SHG are very different nonlinear processes, it is instructive to compare their spectra measured on 

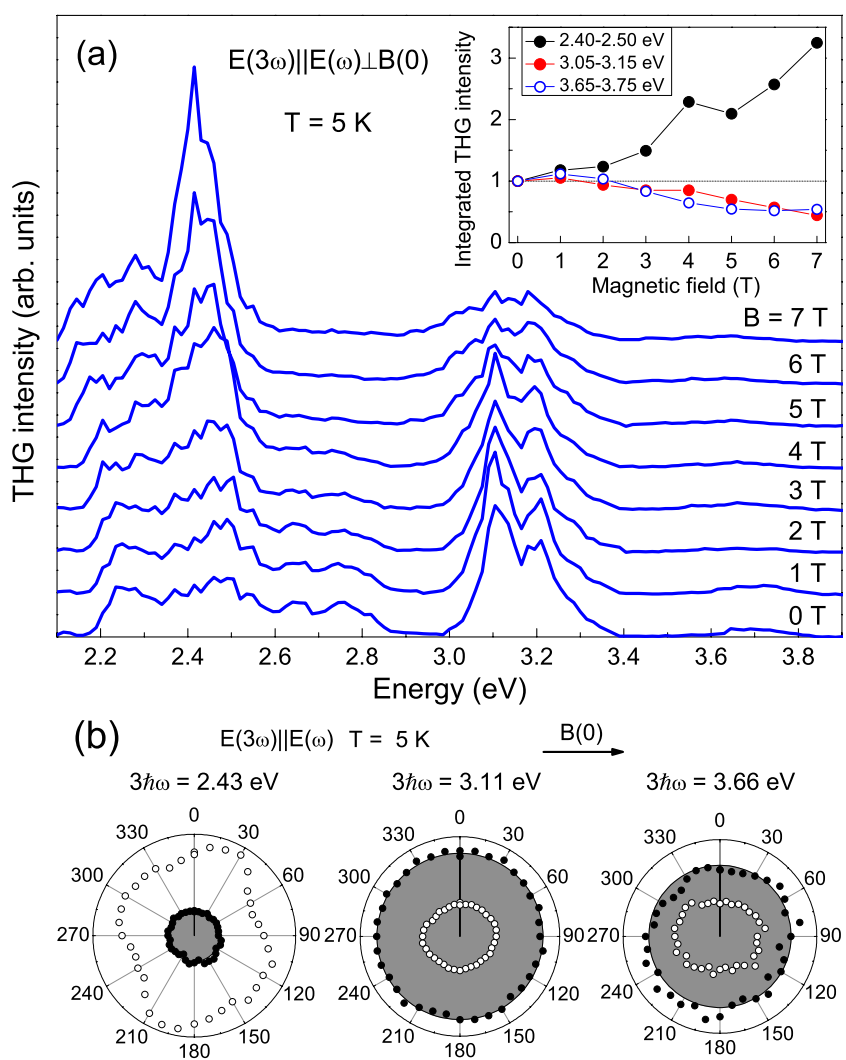

FIG. 7. (Color online) (a) THG spectra of EuTe measured in $\mathbf{E}(3 \omega) \| \mathbf{E}(\omega) \perp \mathbf{B}(0)$ configuration for different magnetic fields at $T=5 \mathrm{~K}$. Inset shows magnetic-field dependences of integrated THG intensity for different bands normalized to the intensities at $B$ $=0 \mathrm{~T}$. (b) Rotational anisotropies for the three bands measured in $\mathbf{E}(3 \omega) \| \mathbf{E}(\omega)$ configuration without (closed circles and gray areas) and with external magnetic field of $7 \mathrm{~T}$ (open circles).

the very same sample. This comparison in the $\mathbf{E}(3 \omega, 2 \omega) \| \mathbf{E}(\omega)$ configuration as well as rotational SHG diagrams are given in Fig. 9. There is a good coincidence of the main bands observed in the THG and SHG spectra, which confirms that both processes reflect the electronic structure of EuTe. The intensity of the THG signal is much stronger than the SHG signal. This comes from the fact that

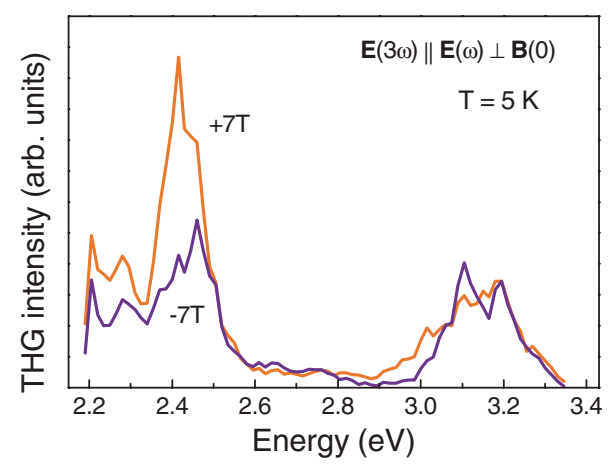

FIG. 8. (Color online) THG spectra of EuTe measured in $\mathbf{E}(3 \omega) \| \mathbf{E}(\omega) \perp \mathbf{B}(0)$ configuration for reversed orientations of external magnetic field of +7 and $-7 \mathrm{~T}$ in the spectral range of 2.2$3.4 \mathrm{eV}$.


FIG. 9. (Color online) (a) SHG and THG spectra of EuTe measured in $\mathbf{E}(3 \omega, 2 \omega) \| \mathbf{E}(\omega) \perp \mathbf{B}(0)$ configuration. The SHG spectrum is enlarged by a factor of two. (b) Rotational diagrams for SHG signals measured at $2 \hbar \omega=2.4 \mathrm{eV}$ for $\mathbf{E}(2 \omega) \| \mathbf{E}(\omega)$ and $\mathbf{E}(2 \omega) \perp \mathbf{E}(\omega)$ configurations at $B=7 \mathrm{~T}$. Experimental data are given by symbols and model fits by filled areas, see text.

for the EuTe crystal structure $m 3 m$ of rocksalt type the SHG is forbidden in electric dipole approximation, but the THG is allowed, which is in line with the symmetry considerations in Sec. III. The SHG in europium chalcogenides $\mathrm{Eu} X(X$ $=\mathrm{O}, \mathrm{S}, \mathrm{Se}$, and $\mathrm{Te}$ ) can be observed only in magnetic dipole approximation. ${ }^{6-8}$ Note that the pure IMD contribution to the SHG signal has a twofold symmetry, ${ }^{7}$ contrary to the fourfold symmetry of the THG signal, compare with Fig. 7(b). Indeed the SHG diagrams in Fig. 9(b) are mostly twofold, some deviation from the twofold symmetry can be related to the strain distortion typical for EuTe layers grown on $\mathrm{BaF}_{2}$ substrates, for more details see Ref. 7. Gray areas represent fits according to Eqs. (12)-(16) from Ref. 7.

\section{DISCUSSION AND A QUANTUM-MECHANICAL MODEL OF THG}

In this section we shall examine a THG model in the framework of the electronic structure which describes well the dichroic band-edge optical absorption, ${ }^{57-59}$ and also the SHG in EuTe. ${ }^{60}$ The main purpose is to verify the adequateness of the level scheme to describe the main features of the THG spectrum, and, in particular, to gain an understanding why the THG efficiency is nonzero in zero magnetic field. This is in contrast to the SHG efficiency, which is zero if no magnetic field is applied to the EuTe crystal. For this purpose 


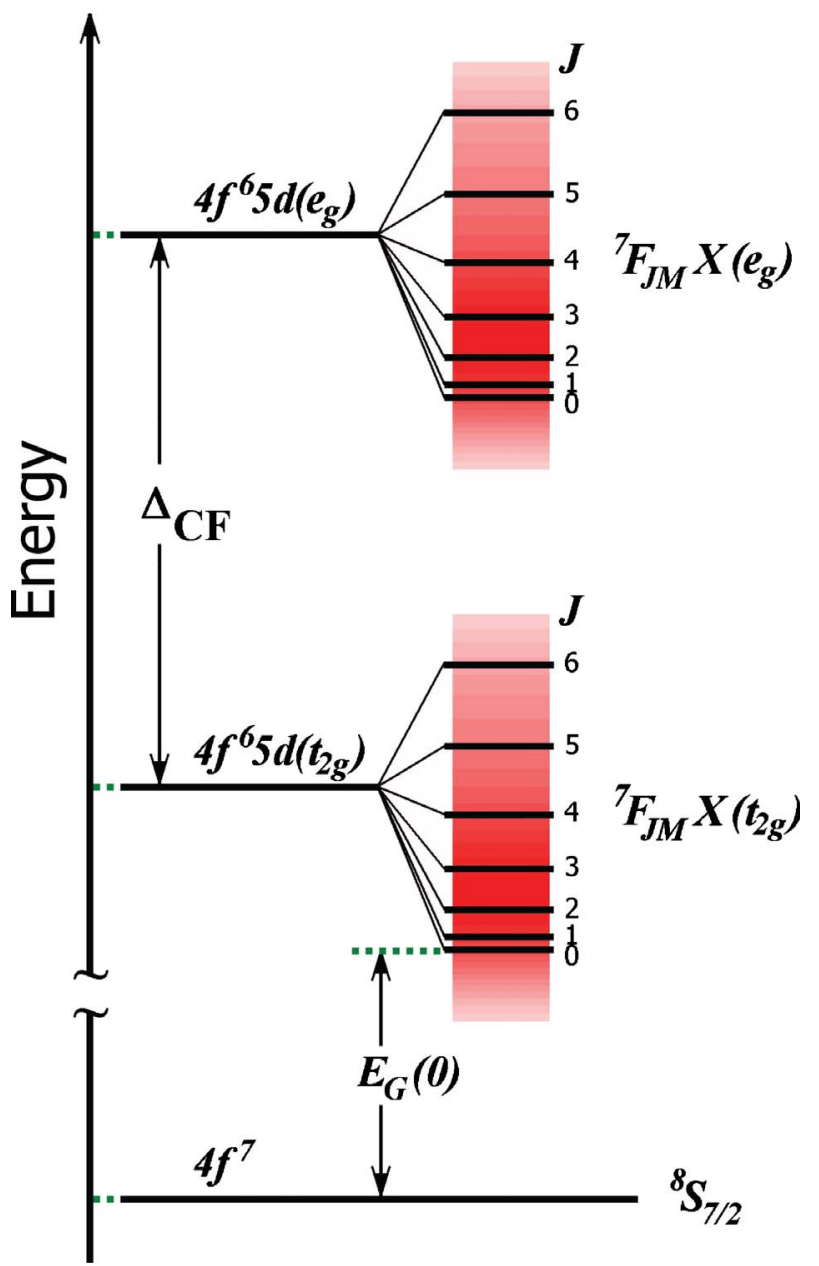

FIG. 10. (Color online) Ground and lowest energy excited states in $\mathrm{EuX}$. The distance between the center of the ${ }^{7} F_{J M} X$ state for $J$ $=0$ and the ${ }^{8} S_{7 / 2}$ state is taken as the band gap $E_{G}(0)$. The splitting in the ${ }^{7} F_{J M}$ manifold is due to the spin-orbit interaction, with a spin-orbit constant $\lambda_{4 f}$. The shaded area indicates the width of the seven overlapping $5 d\left(t_{2 g}\right)$ and $5 d\left(e_{g}\right)$ tight-binding bands.

it is sufficient to examine THG in parallel configuration, $\mathbf{E}(3 \omega) \| \mathbf{E}(\omega)$.

The restricted set of electronic states used in the model is shown in Fig. 10. It consists of the $4 f^{7}\left({ }^{8} S_{7 / 2}\right)$ ground state and excited states contained in the $4 f^{6} 5 d^{1}$ configuration. The excited configuration will henceforward be labeled in spectroscopic notation by ${ }^{7} F_{J M} X$, where ${ }^{7} F_{J M}$ represents the state of the six electrons in the $4 f$ shell at an $\mathrm{Eu}^{3+}$ site, and $X$ represents an electron transferred into a Bloch state. The electric dipole can couple the ground state to the $5 d\left(t_{2 g}\right)$ or $5 d\left(e_{g}\right)$ conduction bands but not to the conduction band of $6 \mathrm{~s}$ character. Therefore, we restrict our analysis to the $5 d\left(t_{2 g}\right)$ and $5 d\left(e_{g}\right)$ conduction-band states, denoted shortly by $X$. In the tight-binding scheme $X$ is given by

$$
X(\boldsymbol{k}, \boldsymbol{r})=\frac{1}{\sqrt{\mathcal{N}}} \sum_{\boldsymbol{R}} e^{i \boldsymbol{R} \cdot \boldsymbol{k}} \phi(\boldsymbol{r}-\boldsymbol{R}),
$$

where $\phi(\boldsymbol{r})$ represents one of the three $5 d\left(t_{2 g}\right)$ europium orbitals $\left(d_{x y}, d_{y z}\right.$, or $\left.d_{z x}\right)$, or one of the two $5 d\left(e_{g}\right)$ europium

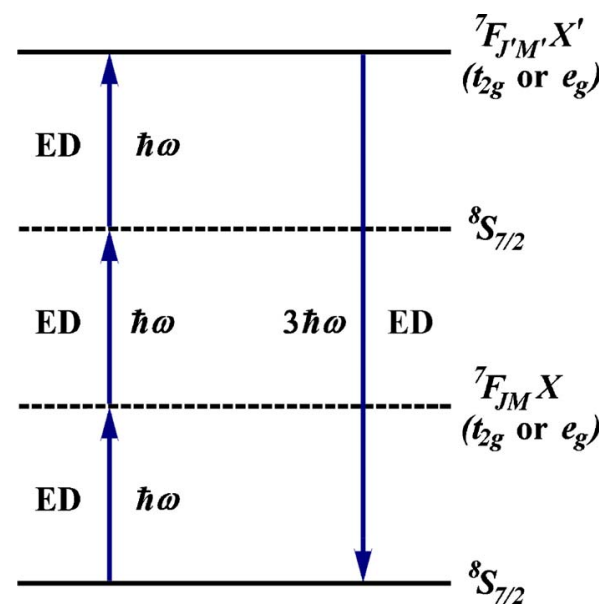

FIG. 11. (Color online) THG scheme in EuTe considered in this work, following the notation of Ref. 10, Sec. 3.1. The solid line at the bottom represents the ground state, ${ }^{8} S_{7 / 2}$, of seven $f$ electrons on a $\mathrm{Eu}^{2+}$ lattice site. The solid line at the top represents an excited state ${ }^{7} F_{J^{\prime} M^{\prime}} X^{\prime}$, consisting of 6 electrons on a $\mathrm{Eu}^{3+}$ site, plus an electron in a $5 d\left(t_{2 g}\right)$ or $5 d\left(e_{g}\right)$ conduction band. The scheme assumes that the three-photon transition is resonant with the energy of the ${ }^{8} S_{7 / 2}-{ }^{7} F_{J^{\prime} M^{\prime}} X^{\prime}$ energy gap (which is approximately the EuTe band gap), thereby resonantly enhancing the efficiency of the THG process. The dashed lines represent the two intermediate (virtual) electronic states involved in the instantaneous four-step process of the THG. The transition into the virtual states is allowed by the electric dipole selection rule, however, it is not resonant with the incident photon energy. Therefore, although energy is conserved in the complete THG process, it is not conserved in separate steps of the THG process.

orbitals $\left(d_{z^{2}}\right.$ or $\left.d_{x^{2}-y^{2}}\right) . \boldsymbol{R}$ is the position vector of an Eu atom in the lattice and $\mathcal{N}$ is the number of lattice sites inside the Born-von Karmán volume.

In the present model, the Zeeman energies of the $\mathrm{Eu}^{2+}$ ions are disregarded because the Zeeman shift of electronic levels is negligibly small in comparison to the giant magnetic-field-dependent energy shift associated with the $d$ - $f$ exchange interaction. Consequently, the ${ }^{7} F_{J M}$ levels have a $2 M+1$-fold degeneracy, and the energy of an electronic excitation, $E_{J X}$, depends only on the quantum numbers $J$ and $X$,

$$
E_{J X}=E_{G}+\Delta_{X}+\frac{1}{2} \lambda_{4 f} J(J+1)+\epsilon_{X}(\boldsymbol{k}), \quad J=0, \ldots, 6,
$$

where $\lambda_{4 f}=9.6 \mathrm{meV}$ is the spin-orbit interaction constant for electrons in the $4 f$ orbital of the $\mathrm{Eu}^{2+}$ ion $^{57}$ and $\epsilon_{X}(\boldsymbol{k})$ is the energy dispersion of the $5 d\left(t_{2 g}\right)$ or $5 d\left(e_{g}\right)$ conduction band; $\Delta_{X}$ is given by

$$
\Delta_{X}=\left\{\begin{array}{cl}
0 & \text { if } X \text { belongs to a } 5 d\left(t_{2 g}\right) \text { band } \\
\Delta_{\mathrm{CF}} & \text { if } X \text { belongs to a } 5 d\left(e_{g}\right) \text { band }
\end{array}\right.
$$

$E_{G}$ is the magnetic-field-dependent energy band gap of the $\mathrm{Eu} X$ system, which for EuTe is given by ${ }^{58}$ 


$$
E_{G}=E_{G}(0)-J_{d f} S\left\{\begin{array}{cl}
\left(\frac{B}{B_{\text {sat }}}\right)^{2} & \text { if } B<B_{\text {sat }} \\
1 & \text { if } B \geq B_{\text {sat }},
\end{array}\right.
$$

where $E_{G}(0)=2.321 \mathrm{eV}$ is the zero-field band gap, and $J_{d f} S$ is the $d$ - $f$ exchange constant, taken to be $J_{d f} S=130 \mathrm{meV}^{60}$ We also assume $T=0 \mathrm{~K}$, meaning that the unperturbed electronic system is in the ground state $4 f^{7}\left({ }^{8} S_{7 / 2}\right)$.

We shall use two characteristics of the above described electronic energy level scheme: (i) the energy spread of the excited states is much smaller than the band gap, i.e.,
$E_{G} \gg \lambda_{4 f}$ and $E_{G}$ is much larger than the energy width of $\epsilon_{X}(\boldsymbol{k})$; (ii) all excited states have the same parity, which is opposite to the parity of the ground state. From the first property, it can be concluded that the dominant third-order induced polarization will be strongly resonant when the photon energy is about $\hbar \omega \sim \frac{1}{3}\left(E_{G}+\Delta_{X}\right)$. Therefore, we may discard the antiresonant contributions ${ }^{9,10}$ to the induced thirdorder dipole moment. From the second property, electric dipole matrix elements between excited states vanish. Consequently, for photon energies of about a third of the band gap, $\hbar \omega \sim \frac{1}{3}\left(E_{G}+\Delta_{X}\right)$, the third-order polarization amplitude $P_{x 0}^{(3)}(3 \omega)$ can be approximated by

$$
P_{x 0}^{(3)}(3 \omega)=\frac{N e^{4} E_{x}^{3}}{2(2 \hbar \omega)^{3}} \sum_{J M X, J^{\prime} M^{\prime} X^{\prime}} E_{J}^{2} E_{J^{\prime}} \frac{\left|\left\langle{ }^{8} S_{7 / 2}\left|\sum_{i=1}^{7} x_{i}\right|^{8} F_{J M} X\right\rangle\right|{ }^{2}\left|\left\langle{ }^{8} S_{7 / 2}\left|\sum_{i=1}^{7} x_{i}\right|^{8} F_{J^{\prime} M^{\prime}} X^{\prime}\right\rangle\right|^{2}}{\left(E_{J^{\prime} X^{\prime}}-\hbar \omega\right)(0-2 \hbar \omega)\left(E_{J X}-3 \hbar \omega\right)},
$$

where $E_{x}$ is the amplitude of the electric field of the incident light, $x_{i}$ denotes the $i$ th electron position vector projected onto the polarization vector of the exciting light. The THG process associated with Eq. (19) is shown schematically in Fig. 11. For the sake of simplicity, we have only considered THG for the case that both $X$ and $X^{\prime}$ belong to the same crystal-field split conduction band [either $5 d\left(t_{2 g}\right)$ or $5 d\left(e_{g}\right)$ ].

The matrix elements in Eq. (19) involve seven electrons and can be converted into single-electron matrix elements by taking into account spin conservation..$^{57,59}$ This is done by performing a Clebsch-Gordan expansion of the ${ }^{7} F_{J M}$ states, which is then represented by a series of terms with defined electronic spin. The final result for $P_{x 0}^{(3)}(3 \omega)$ is

$$
\begin{aligned}
P_{x 0}^{(3)}(3 \omega)= & \frac{N e^{4} E_{x}^{3}}{2(2 \hbar \omega)^{3}} \sum_{J X, J^{\prime} X^{\prime}} E_{J}^{2} E_{J^{\prime}} \\
& \times \frac{\sum_{M M^{\prime}}\left|C^{J M}\langle 4 f m|x| X\rangle\right|^{2}\left|C^{J^{\prime} M^{\prime}}\left\langle 4 f m^{\prime}|x| X^{\prime}\right\rangle\right|^{2}}{\left(E_{J^{\prime} X^{\prime}}-\hbar \omega+i \Gamma\right)(\hbar \omega+i \Gamma)\left(E_{J X}-3 \hbar \omega+i \Gamma\right)},
\end{aligned}
$$

where $m=-M+3$ and $m^{\prime}=-M^{\prime}+3$, the $|4 f m\rangle$ represent single-electron $4 f$ orbitals of the $\mathrm{Eu}^{2+}$ ion, and $C^{J M}$ $=\left(L S M_{L} M_{S} \mid J M\right)$ is a compact representation of the ClebschGordan coefficients with $L=S=M_{S}=3, M_{L}=M-3$. Here we have introduced the constant $\Gamma$, which is the characteristic energy uncertainty of the single-particle energy levels associated with their finite lifetimes. ${ }^{9,10}$

To calculate the THG spectrum, we shall take advantage of the narrowness of the conduction band, and the nonconservation of the Bloch wave vector $\boldsymbol{k}$ in the matrix elements of Eq. (20). The latter is a consequence of the spatial localization of the ground-state electrons. Therefore, the conduction-band energy width will lead to broadening of the resonances that are characteristic of Eq. (20). The THG spectrum is calculated as follows. In a first step, the conductionband dispersion is ignored, and Eq. (20) is calculated using $\phi$ and $\phi^{\prime}$ orbitals of $5 d\left(t_{2 g}\right)$ or $5 d\left(e_{g}\right)$ symmetry, localized at a fixed lattice site. Then, calculation of all matrix elements, such as $\langle 4 f m|x| \phi\rangle$, can be done as described in detail in Ref. 60.

The width of the conduction-band states, which was ignored in the first calculation step, as described above, is taken into account in a second step, by convolving the thirdorder polarization with a density of states $\mathcal{D}(E)$,

$$
I_{\mathrm{THG}}(3 \hbar \omega) \propto \int d E \mathcal{D}(E-3 \hbar \omega)\left\{\operatorname{Re}\left[P_{x 0}^{(3)}\left(\frac{E}{\hbar}\right)\right]\right\}^{2} .
$$

Henceforward we choose a heuristic approach in which $\mathcal{D}(E)$ is taken to be a sum of Gaussian functions centered at the energies $E_{J X}$ [Eq. (16)]. In order to keep the theoretical model as simple as possible and tractable, the full width of all Gaussians were fixed at a common adjustable value $\delta$.

The THG calculation requires knowledge of the spatial orientation of the $\mathrm{Eu}^{2+}$ spins in the crystal lattice, which is done using molecular-field theory. ${ }^{61}$ At $B=0$ the $\mathrm{Eu}^{2+}$ spins lie on the (111) planes. Since there are four equivalent (111) planes, four so-called $\mathrm{T}$ domains are formed. Within each $\mathrm{T}$ domain, the $\mathrm{Eu}^{2+}$ spins can point along any of the three equivalent $[11 \overline{2}]$ directions, hence each $\mathrm{T}$ domain contains three so-called S domains. The amplitude of the third-order induced polarization generated by a separate magnetic domain was calculated using Eq. (20). The total polarization was obtained by averaging over all magnetic domains and both spin sublattices. The THG intensity was calculated using Eq. (21). A sample containing a single $\mathrm{T}$ domain was 


\section{(a) $B=0$}


\section{(b) $B_{\mathrm{SAT}} \|[\overline{1} 10]$}
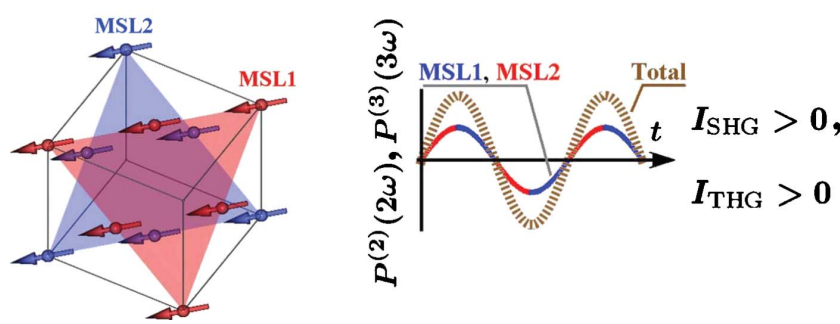

FIG. 12. (Color online) Schematic representation of the SHG and THG processes in EuTe. (a) At $B=0$, the lattice spins in the two magnetic sublattices (MSL1 and MSL2) are antiparallel; as a result, the second-order polarizations from both sublattices cancel each other, and no SHG occurs. In contrast, the third-order polarizations from both sublattices are always in phase with one another and the THG process operates at all magnetic fields. (b) At $B$ corresponding to the saturation value, causing ferromagnetic alignment of both sublattices, the SHG process is allowed, as is the THG process.

assumed, because it is known that in EuTe epitaxial layers, grown by MBE, a single $\mathrm{T}$ domain is present. ${ }^{62}$

Although the Zeeman energies do not enter the energy level scheme, an external magnetic field still has a fundamental effect on the SHG and THG efficiencies in EuX, because the strength and direction of the magnetic field determines the orientation of the $\mathrm{Eu}^{2+}$ spins in the lattice. ${ }^{61}$ For example, as illustrated in Fig. 12(a), for zero magnetic field, the order in EuTe is antiferromagnetic. SHG is due to the magnetic dipole coupling between excited states, ${ }^{6,7,60}$ and the polarization waves arising from both magnetic sublattice, containing $\mathrm{Eu}^{2+}$ spins pointing in opposite directions, are in opposite phase to each other, and their interference leads to a zero net second-order polarization. Thus SHG vanishes in zero field. When the applied magnetic field aligns all $\mathrm{Eu}^{2+}$ spins ferromagnetically, as shown in Fig. 12(b), a single magnetic sublattice remains, and SHG becomes efficient.

In contrast to the SHG process, in which the virtual and resonant states are coupled through the magnetic dipole, in the THG process the virtual and resonant states are coupled by the electric dipole operator. As a consequence of this, the third-order polarization waves arising from distinct magnetic sublattices can interfere constructively even in zero magnetic field, when the spin order is antiferromagnetic, as illustrated in Fig. 12(a). As described in the paragraph following Eq. (20), our model disregards THG processes involving crossed transitions ${ }^{7} F_{J M} X \rightarrow{ }^{7} F_{J^{\prime} M^{\prime}} X^{\prime}$, when $X$ and $X^{\prime}$ are of different symmetries, consequently, for the $\mathbf{E}(3 \omega) \| \mathbf{E}(\omega)$ geometry examined in this section, only the absolute values of the matrix elements enter Eq. (20). This means that at a given frequency, the third-order polarization waves coming from all spin orientations are in phase with one another, and always interfere constructively, as illustrated in Figs. 12(a) and 12(b). Nevertheless, the THG intensity will still strongly depend on the intensity of the external magnetic field and its orientation relative to the crystallographic axes, because the magnetic-field vector determines the orientation of spins in the lattice, which in turn determines the magnitude of the matrix elements appearing in Eq. (20)

In summary, the simplified model used in this section describes THG that is nonzero at $B=0$ but whose spectral contents and intensity still depends on the external magnetic field. The model does not separate the THG into CED (magnetic-field-independent) and IED (magnetic-fielddependent) contributions, which was the general macroscopic approach of Sec. III; instead, in this section we examined THG processes associated with a restricted specific set of electronic levels. The contrast between a nonzero THG intensity and zero SHG, for an antiferromagnetic spin order, is traced to the fact that in the THG process virtual and resonant states are coupled by the electric dipole operator, whereas in the SHG process virtual and resonant states are coupled by the magnetic dipole operator. The absolute value of the THG intensity and its increase, or decrease, with applied magnetic field, is determined by the magnitude of the matrix elements involved in Eq. (20). These matrix elements are strongly dependent on the spatial orientation of the electronic orbitals contained in them; such an orientation is determined by the modulus of the magnetic field, as well as its direction in respect to the crystallographic axes. However, because of the symmetry of the restricted electronic states contained in the model of this section, inverting the magnetic-field direction does not change the calculated THG intensity.

The THG spectra for EuTe were calculated using wellknown parameters characteristic of its electronic structure: ${ }^{60}$ $E_{G}=2.321 \mathrm{eV}, J_{d f} S=0.13 \mathrm{eV}, \lambda_{4 f}=9.6 \mathrm{meV}$, and the splitting between $t_{2 g}$ and $e_{g}$ bands $\Delta_{\mathrm{CF}} \sim 1$ (see Fig. 2). The adjustable parameters were $\delta$, which determines the approximated density of states in Eq. (21) and the level width $\Gamma$, fixed at $50 \mathrm{meV}$ and $2 \mathrm{meV}$, respectively.

Calculated THG spectra as a function of magnetic field are shown in Fig. 13 and can be compared with the experimental result shown in Fig. 7(a). A comparison of Fig. 13 and Fig. 7(a) shows that the calculated spectra contain emission bands around $2.4 \mathrm{eV}$ and $3.15 \mathrm{eV}$, in agreement with experiment, demonstrating that these emissions can be associated with the $t_{2 g}$ and $e_{g}$ conduction bands, respectively. The redshift with increasing magnetic field of the THG emissions is also reproduced by the theory, as well as the sharpening of the $t_{2 g}$ peak when $B$ increases. Important to note that the absence of rotational anisotropy of the $t_{2 g}$ emission expected theoretically (see Fig. 14) also agrees with the experimental result (inset in Fig. 4). The main discrepancy between theory and experiment concerns the $e_{g}$ THG emission, which at $B$ $=0$ is experimentally stronger than the $t_{2 g}$ one, whereas the opposite is seen in the theoretical spectrum. An inaccurate 




FIG. 13. (Color online) Calculated THG spectra in EuTe at $T$ $=0 \mathrm{~K}$. Calculations were done for $\mathbf{E}(3 \omega) \| \mathbf{E}(\omega) \perp \mathbf{B}(0)$ configuration, $\mathbf{k} \|[111]$ and $\mathbf{B} \|[\overline{1} 10]$. Thin (blue) lines show the unbroadened spectra, thick black lines show the broadened spectra as given by Eq. (21).

description of the $e_{g}$ THG process by our model is not at all surprising, because the theory uses the tight-binding approximation, which is much more appropriate to the less energetic $t_{2 g}$ state. An improved description of the THG process in the whole energy range would require a density of states beyond the simplified tight-binding model used, which is not justifiable, because it would complicate still further an already complex model. It must be emphasized that in order to keep the model tractable, the following simplifications were made: (1) only the ${ }^{8} S_{7 / 2}$ and ${ }^{7} F_{J M} X$ electronic levels were assumed to be optically active; (2) the tight-binding approximation with a common broadening parameter $\delta$ for all levels was used. Although these approximations can be quite acceptable to describe the $t_{2 g}$ conduction band, they can only provide a very crude description of the more energetic $e_{g}$ states. Nevertheless, despite its limitations, the theoretical model still serves to demonstrate that the THG spectra can be associated with $t_{2 g}$ states and, at least crudely, to $e_{g}$ states; it describes reasonably well many of the observed THG characteristics, and it provides a clear description of the basic differences between SHG and THG processes in EuTe.

\section{CONCLUSIONS}

We performed third-harmonic spectroscopy for the magnetic semiconductor EuTe in external magnetic field and observed several resonances in the vicinity of the band gap at

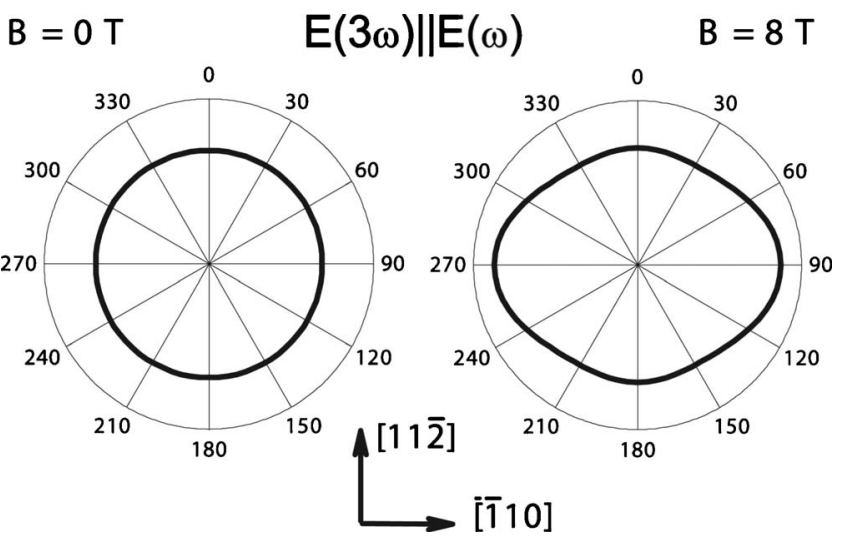

FIG. 14. Calculated THG anisotropy for the integrated intensity of the $5 d\left(t_{2 g}\right)$ optical transition in EuTe at $T=0 \mathrm{~K}$. Calculations were performed for $\mathbf{E}(3 \omega) \| \mathbf{E}(\omega)$ configuration and $\mathbf{B} \|[\overline{1} 10]$.

2.2-2.5 eV and at higher energies up to $4 \mathrm{eV}$, which can be associated with four-photon THG processes. We have shown that particular features of the crystallographic structure and the electronic band structure of the centrosymmetric EuTe allow crystallographic and magnetic-field-induced THG. In contrast to the SHG studies, ${ }^{6,7,60}$ THG of electric dipole type was observed in zero magnetic field. The contained resonances were assigned to specific electronic transitions between the ground $4 f^{7}$ state at the top of the valence band and excited $4 f^{6} 5 d^{1}$ states of the $\mathrm{Eu}^{2+}$ ions, which form the lowest energy conduction band. A strong modification of the THG intensity was observed in applied magnetic fields at $2.4 \mathrm{eV}$, which gives an unambiguous proof of the magnetic-fieldinduced contribution to the THG. Two main features of the THG were assigned to $5 d\left(t_{2 g}\right)$ and $5 d\left(e_{g}\right)$ subbands at $2.4 \mathrm{eV}$ and $3.15 \mathrm{eV}$, respectively. Temperature, magnetic-field, and rotational anisotropy studies allowed us to distinguish between crystallographic and magnetic-field-induced contributions to the THG. A microscopic quantum-mechanical model of the THG response was developed, which provides an electronic level scheme basis for the experimental observations and explains the differences between THG and SHG mechanisms in EuTe. We foresee that THG spectroscopy can be extended to many other centrosymmetric magnetic bulk semiconductors and insulators, as well as to thin films and artificial structures, where SHG processes of electric dipole type are forbidden, but THG processes are allowed.

\section{ACKNOWLEDGMENTS}

This work was supported by the Deutsche Forschungsgemeinschaft (Grant No. YA65/4-1), the Russian Foundation for Basic Research, Russian Academy Programs on Spintronics, Physics of Nanostructures, Quantum Physics of Condensed Matter; FoNE of the European Science Foundation, the Brazilian Agencies FAPESP and CNPq; the Deutscher Akademischer Austauschdienst (DAAD), and the FWF (I80N20, Vienna, Austria). 
${ }^{1} \mathrm{P}$. Wachter, in Handbook on the Physics and Chemistry of Rare Earths, edited by K. A. Gschneider and L. R. Eyring (NorthHolland, Amsterdam, 1979), Vol. 11, p. 507.

${ }^{2}$ G. Güntherodt, Z. Phys. B: Condens. Matter 18, 37 (1974).

${ }^{3}$ E. L. Nagaev, Physics of Magnetic Semiconductors (Mir, Moscow, 1983).

${ }^{4}$ A. Schmehl, V. Vaithyanathan, A. Herrnberger, S. Thiel, C. Richter, M. Liberati, T. Heeg, M. Rockerath, L. F. Kourkoutis, S. Muhlbauer, P. Boni, D. A. Muller, Y. Barash, J. Schubert, Y. Idzerda, J. Mannhart, and D. G. Schlom, Nature Mater. 6, 882 (2007).

${ }^{5}$ G. Springholz, A. Raab, R. T. Lechner, and V. Holy, Appl. Phys. Lett. 79, 1225 (2001).

${ }^{6}$ B. Kaminski, M. Lafrentz, R. V. Pisarev, D. R. Yakovlev, V. V. Pavlov, V. A. Lukoshkin, A. B. Henriques, G. Springholz, G. Bauer, E. Abramof, P. H. O. Rappl, and M. Bayer, Phys. Rev. Lett. 103, 057203 (2009).

${ }^{7}$ B. Kaminski, M. Lafrentz, R. V. Pisarev, D. R. Yakovlev, V. V. Pavlov, V. A. Lukoshkin, A. B. Henriques, G. Springholz, G. Bauer, E. Abramof, P. H. O. Rappl, and M. Bayer, Phys. Rev. B 81, 155201 (2010).

${ }^{8}$ M. Matsubara, A. Schmehl, J. Mannhart, D. G. Schlom, and M. Fiebig, Phys. Rev. B 81, 214447 (2010).

${ }^{9}$ Y. R. Shen, The Principles of Nonlinear Optics (Wiley, New York, 1984).

${ }^{10}$ R. W. Boyd, Nonlinear Optics (Academic, San Diego, 1993).

${ }^{11}$ M. Fiebig, V. V. Pavlov, and R. V. Pisarev, J. Opt. Soc. Am. 22, 96 (2005).

${ }^{12}$ D. N. Nikogosyan, Nonlinear Optical Crystals: A Complete Survey (Springer, Berlin, 2005).

${ }^{13}$ S. V. Popov, Y. P. Svirko, and N. I. Zheludev, Susceptibility Tensors for Nonlinear Optics (Institute of Physics, Philadelphia, 1995).

${ }^{14}$ R. R. Birss, Symmetry and Magnetism (North-Holland, Amsterdam, 1967).

${ }^{15}$ S. A. Akhmanov, V. I. Emel'yanov, N. I. Koroteev, and V. N. Seminogov, Sov. Phys. Usp. 28, 1084 (1985).

${ }^{16}$ A. Schülzgen, Y. Kawabe, E. Hanamura, A. Yamanaka, P.-A. Blanche, J. Lee, H. Sato, M. Naito, N. T. Dan, S. Uchida, Y. Tanabe, and N. Peyghambarian, Phys. Rev. Lett. 86, 3164 (2001).

${ }^{17}$ V. V. Pavlov, R. V. Pisarev, M. Fiebig, and D. Fröhlich, Low Temp. Phys. 28, 523 (2002).

${ }^{18}$ V. V. Pavlov, R. V. Pisarev, M. Fiebig, and D. Fröhlich, Phys. Solid State 45, 662 (2003).

${ }^{19}$ J. Shimura, Sh.-i. Ohkoshi, and K. Hashimoto, Appl. Phys. Lett. 82, 3290 (2003).

${ }^{20}$ O. A. Aktsipetrov, T. V. Murzina, E. M. Kim, R. V. Kapra, A. A. Fedyanin, M. Inoue, A. F. Kravets, S. V. Kuznetsova, M. V. Ivanchenko, and V. G. Lifshits, J. Opt. Soc. Am. B 22, 138 (2005).

${ }^{21}$ Sh.-i. Ohkoshi, J. Shimura, K. Ikeda, and K. Hashimoto, J. Opt. Soc. Am. B 22, 196 (2005).

${ }^{22}$ O. A. Aktsipetrov, T. V. Dolgova, A. A. Fedyanin, T. V. Murzina, M. Inoue, K. Nishimura, and H. Uchida, J. Opt. Soc. Am. B 22, 176 (2005).

${ }^{23}$ O. Masihzadeh, Ph. Schlup, and R. A. Bartels, Opt. Lett. 34, 1240 (2009).

${ }^{24}$ J. Squier, M. Muller, G. Brakenhoff, and K. R. Wilson, Opt. Express 3, 315 (1998).
${ }^{25}$ D. Yelin and Y. Silberberg, Opt. Express 5, 169 (1999).

${ }^{26}$ E. J. Gualda, G. Filippidis, K. Melessanaki, and C. Fotakis, Appl. Spectrosc. 63, 280 (2009).

${ }^{27}$ V. V. Pavlov, A. M. Kalashnikova, R. V. Pisarev, I. Sänger, D. R. Yakovlev, and M. Bayer, Phys. Rev. Lett. 94, 157404 (2005).

${ }^{28}$ I. Sänger, D. R. Yakovlev, R. V. Pisarev, V. V. Pavlov, M. Bayer, G. Karczewski, T. Wojtowicz, and J. Kossut, Phys. Rev. Lett. 96, 117211 (2006).

${ }^{29}$ N. Van tran, J. H. McFee, and C. K. N. Patel, Phys. Rev. Lett. 21, 735 (1968).

${ }^{30}$ S. E. Mani, J. I. Jang, and J. B. Ketterson, Opt. Lett. 34, 2817 (2009).

${ }^{31}$ P. G. Steeneken, L. H. Tjeng, I. Elfimov, G. A. Sawatzky, G. Ghiringhelli, N. B. Brookes, and D.-J. Huang, Phys. Rev. Lett. 88, 047201 (2002).

${ }^{32}$ T. S. Santos and J. S. Moodera, Phys. Rev. B 69, 241203 (2004).

${ }^{33}$ T. S. Santos, J. S. Moodera, K. V. Raman, E. Negusse, J. Holroyd, J. Dvorak, M. Liberati, Y. U. Idzerda, and E. Arenholz, Phys. Rev. Lett. 101, 147201 (2008).

${ }^{34}$ G.-X. Miao, M. Müller, and J. S. Moodera, Phys. Rev. Lett. 102, 076601 (2009)

${ }^{35}$ J. S. Moodera, X. Hao, G. A. Gibson, and R. Meservey, Phys. Rev. Lett. 61, 637 (1988).

${ }^{36}$ X. Hao, J. S. Moodera, and R. Meservey, Phys. Rev. B 42, 8235 (1990).

${ }^{37}$ A. T. Filip, P. LeClair, C. J. P. Smits, J. T. Kohlhepp, H. J. M. Swagten, B. Koopmans, and W. J. M. de Jonge, Appl. Phys. Lett. 81, 1815 (2002)

${ }^{38}$ P. LeClair, J. K. Ha, H. J. M. Swagten, J. T. Kohlhepp, C. H. V. de Vin, and W. J. M. de Jonge, Appl. Phys. Lett. 80, 625 (2002).

${ }^{39}$ C. J. P. Smits, A. T. Filip, J. T. Kohlhepp, H. J. M. Swagten, B. Koopmans, and W. J. M. de Jonge, J. Appl. Phys. 95, 7405 (2004).

${ }^{40}$ J. Trbovic, C. Ren, P. Xiong, and S. von Molnar, Appl. Phys. Lett. 87, 082101 (2005).

${ }^{41}$ C. Ren, J. Trbovic, R. L. Kallaher, J. G. Braden, J. S. Parker, S. vonMolnar, and P. Xiong, Phys. Rev. B 75, 205208 (2007).

${ }^{42}$ J. S. Moodera, R. Meservey, and X. Hao, Phys. Rev. Lett. 70, 853 (1993).

${ }^{43}$ J. Lettieri, V. Vaithyanathan, S. K. Eah, J. Stephens, V. Sih, D. D. Awschalom, J. Levy, and D. G. Schlom, Appl. Phys. Lett. 83, 975 (2003).

${ }^{44}$ T. Kasuya, Crit. Rev. Solid State Mater. Sci. 3, 131 (1972).

${ }^{45}$ T. Kasuya, IBM J. Res. Dev. 14, 214 (1970).

${ }^{46}$ A. Mauger and C. Godart, Phys. Rep. 141, 51 (1986).

${ }^{47}$ R. T. Lechner, G. Springholz, T. U. Schülli, J. Stangl, T. Schwarzl, and G. Bauer, Phys. Rev. Lett. 94, 157201 (2005).

${ }^{48}$ J. Ferré, J. Phys. (France) 35, 781 (1974).

${ }^{49}$ J. Schoenes and P. Wachter, IEEE Trans. Magn. 12, 81 (1976).

${ }^{50} \mathrm{G}$. Busch, J. Schoenes, and P. Wachter, Solid State Commun. 8, 1841 (1970).

${ }^{51}$ J. Schoenes and P. Wachter, Physica B 86-88, 125 (1977).

${ }^{52}$ M. Suekane, G. Kido, N. Miura, and S. Chikazumi, J. Magn. Magn. Mater. 31-34, 589 (1983).

${ }^{53}$ H. Hori, R. Akimoto, M. Kobayashi, S. Miyamoto, M. Furusawa, N. Kreines, A. Yamagishi, and M. Date, Physica B 201, 438 (1994).

${ }^{54}$ M. R. Koblischka and R. J. Wijngaarden, Supercond. Sci. Technol. 8, 199 (1995).

${ }^{55}$ R.-P. Pan, H. D. Wei, and Y. R. Shen, Phys. Rev. B 39, 1229 
(1989).

${ }^{56}$ W. Heiss, G. Prechtl, and G. Springholz, Phys. Rev. B 63, 165323 (2001).

${ }^{57}$ A. B. Henriques, A. Wierts, M. A. Manfrini, G. Springholz, P. H. O. Rappl, E. Abramof, and A. Y. Ueta, Phys. Rev. B 72, 155337 (2005).

${ }^{58}$ L. K. Hanamoto, A. B. Henriques, N. F. Oliveira, Jr., P. Rappl, E. Abramof, and Y. Ueta, J. Phys.: Condens. Matter 16, 5597 (2004).
${ }^{59}$ A. B. Henriques, M. A. Manfrini, P. H. O. Rappl, and E. Abramof, Phys. Rev. B 77, 035204 (2008).

${ }^{60}$ A. B. Henriques, E. Abramof, and P. H. O. Rappl, Phys. Rev. B 80, 245206 (2009).

${ }^{61}$ A. B. Henriques, G. D. Galgano, B. Díaz, P. H. O. Rappl, and E. Abramof, J. Phys.: Condens. Matter 19, 406234 (2007).

${ }^{62}$ B. Díaz, E. Granado, E. Abramof, P. H. O. Rappl, V. A. Chitta, and A. B. Henriques, Phys. Rev. B 78, 134423 (2008). 